\title{
Beans Quality Inspection Using Correlation-Based Granulometry
}

Sidnei Alves de Araújo ${ }^{1,2^{*}}$, Jorge Henrique Pessota ${ }^{2}$ and Hae Yong Kim ${ }^{1}$

${ }^{1}$ Escola Politécnica, Universidade de São Paulo,

Av. Prof. Luciano Gualberto, tr. 3, 158, 05508-010, São Paulo, SP, Brazil.

${ }^{2}$ Industrial Engineering Post-Graduation Program, Universidade Nove de Julho, Av. Francisco Matarazzo, 612, Água Branca, 05001-100, São Paulo, SP, Brazil.

E-mails: saraujo@uninove.br, jorgepessota@uninove.edu.br, hae@lps.usp.br

*Corresponding author

\begin{abstract}
Bean constitutes, with rice, the staple diet of the Brazilian people. The quality control of beans includes computing the percentages of different varieties present in a batch of beans. The selling price of the batch depends on these percentages. In this work, we propose a computer system for visual inspection of beans. We use "correlation-based multi-shape granulometry" for the first time to spatially localize each grain in the image, together with its size, eccentricity and rotation angle. Using this technique, our system localized correctly 29,993 grains out of 30,000, even in images where many grains were "glued" together. This is the main contribution of our work, because usually other systems fail to individualize "glued" grains. Probably, the same technique can be used in many other agricultural product inspection systems to segment seeds and grains. After segmenting the grains, the system classifies each grain as one of the three most consumed varieties in Brazil, using a technique based on $k$-means and $k$-NN algorithms. This module classified correctly 29,956 grains out of 29,993. These extremely high success rates indicate that proposed system can actually be applied in automated inspection of beans.
\end{abstract}

Keywords: Beans, Granulometry, Classification, Visual Inspection, Computer Vision, Pattern Recognition. 


\section{Introduction}

\subsection{Automated agricultural products inspection}

The use of automated systems for inspection of agricultural products has increased in recent decades. In the literature, there are many computational tools to control the quality of food and agricultural products. We can cite the quality inspection of fruits and vegetables (Blasco et al. 2009a, 2009b; Savakar and Anami, 2009; Mendoza and Aguilera, 2010; Liu et al., 2011; Savakar, 2012; Rodríguez-Pulido et al., 2013); analysis and classification of seeds and grains (Kiliç et al., 2007; Venora et al., 2007; Carrillo and Peñaloza, 2009; Savakar and Anami, 2009; Venora et al., 2009; Aggarwal and Mohan, 2010; Anami and Savakar, 2010; Laurent et al., 2010; Razavi et al., 2010; Patil et al., 2011; GómezSanchis et al., 2012, Stegmayer et al., 2013) and inspection of products of animal origin (Ticay-Rivas et al., 2013; Saraswat and Arya, 2013).

Despite the increasing importance of automated processes, the usual method for quality inspection of food and agricultural products continues to be the human inspection. Manual inspection is a time-consuming and high-cost task. It is very difficult to standardize the manual inspection (Kiliç et al., 2007). Moreover, the human detection capabilities can be affected by environment and personal factors (Pesante-Santana and Woldstad, 2000; Patil et al., 2011).

\subsection{Bean inspection in Brazil}

Bean is a legume rich in protein and energy. It constitutes, with rice, the staple diet of the Brazilian people. The quality control of this product in Brazil follows a set of standards and procedures of the Brazilian Ministry of Agriculture, Livestock and Supply (BMALS, 2011). 
We describe briefly this procedure. A sample of $250 \mathrm{~g}$ of a batch of beans is separated for moisture analysis and another sample of $250 \mathrm{~g}$ is separated for computing the weight percentage of foreign matters, impurities and dead insects. The quality inspection also looks for defective grains in the sample, such as broken, moldy, burned, crushed, damaged by insects (chopped), sprouted, wrinkled, stained, discolored and damaged by various other causes. The beans of groups I and II (the most consumed by the Brazilian people) can have three ratings numbered 1 to 3 , according to the quantities of defects.

The quality control also computes the percentage of grains of different groups, classes and subclasses present in the sample. The group refers to the botanical species. In Brazil, there are two major groups: Group I comprises the specie Phaseolus vulgaris and Group II the specie Vigna unguiculata. In this work, we will deal only with the Group I,that comprises the most consumed beans in Brazil.

A Group is subdivided into classes and subclasses according to the skin color of the grains. Group I is subdivided into classes White, Black and Colored. The class White is further subdivided into subclasses such as Brancão, Branca, Fradinho, etc. The class Colored is further subdivided into subclasses such as Carioca, Mulatto, Canapu, Corujinha, Azulão, Manteiga, Verde, Vinagre, etc.

In Group I, a batch of beans must contain no more than $3 \%$ of grains of nonpredominant class and no more than $10 \%$ of grains of non-predominant subclass. For example, for a batch of beans to be labeled as Black, it must contain no more than $3 \%$ of White or Colored grains. For a batch of beans to be labeled Carioca, it must contain no more than $3 \%$ of White or Black grains, but it can contain up to $10 \%$ of Mulatto grains, because Carioca and Mulatto are two subclasses of the same Colored class. 
The classification of grains is so important that several institutions in Brazil offer courses for grain classifiers, with a specific module for beans. For example, the link below points to the announcement of a course for classifiers, offered by a university together with the ministry of education ${ }^{1}$. Consequently, a computer vision system that counts automatically the number of grains of different classes and subclasses is a very useful tool in practice.

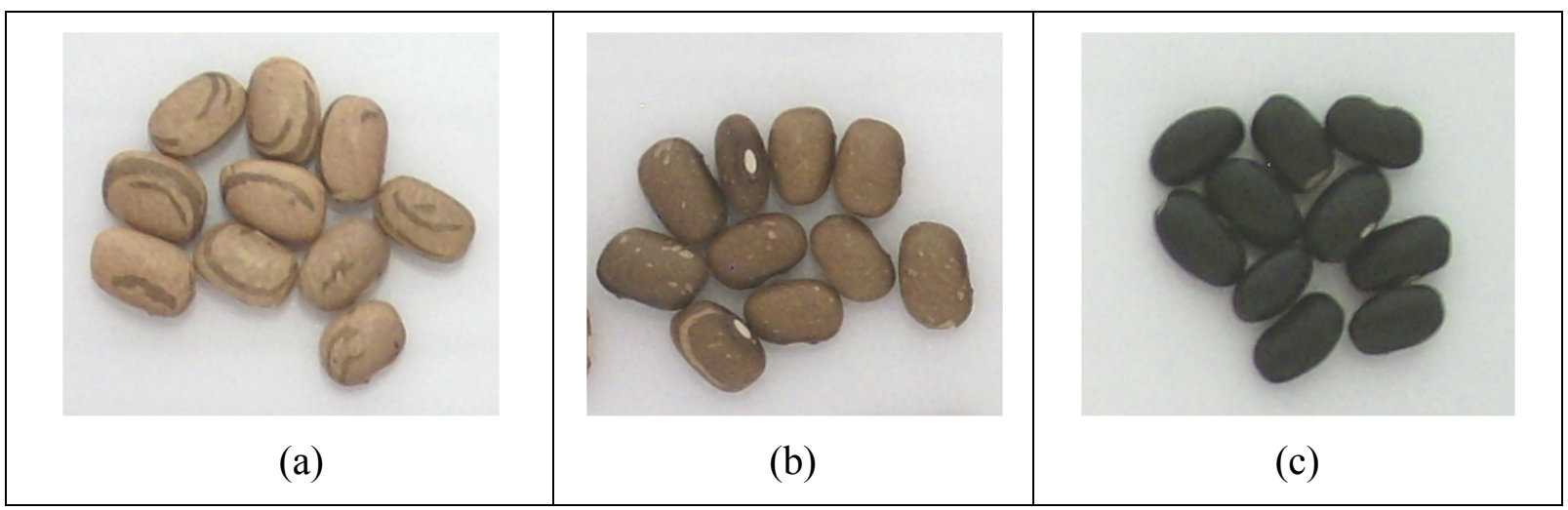

Fig. 1: Samples of most consumed beans in Brazil. (a) Carioca. (b) Mulatto. (c) Black.

\subsection{Automated beans quality inspection}

In the literature there are several works addressing the analysis and classification of seeds and grains. However, we found only five papers on automated systems for classification of beans (Kiliç et al., 2007; Aguilera et al., 2007; Venora et al., 2007, 2009; Laurent et al., 2010). This fact is corroborated by the works of Alfatni et al. (2011) and Patel et al. (2012), where the authors investigated the accuracy of computer vision systems for quality inspection of food and agricultural products.

Kiliç et al. (2007) proposed a computer vision system for beans classification based on the skin colors of the grains. They implemented known techniques such as binarization,

\footnotetext{
${ }^{1}$ http://www.labgraos.com.br/teaser/curso-de-forma $\% \mathrm{C} 3 \% \mathrm{~A} 7 \% \mathrm{C} 3 \% \mathrm{~A} 30$-de-classificadores.pdf
} 
edge detection, mathematical morphology operators and color features quantification by statistical moment in Matlab software. They employed a Multilayer Perceptron for the classification task, obtaining a success rate of $90.6 \%$.

Aguilera el al. (2007) developed a computer vision system to classify seeds, beans, and grains. However, in this paper they only conducted experiments using different types of rice and lentils grains.

Venora et al. (2007) proposed a system for classification of six landraces of beans from Italy, using image analysis library KS-400. In the experiments, they used features like size, shape, color and texture of the grains and obtained success rate of $99.56 \%$. The same authors, in the subsequent work (Venora et al., 2009), conducted new experiments taking into account fifteen Italian traditional landraces of beans, where they achieved a success rate of $98.49 \%$

Laurent et al. (2010) used color histograms and statistical analysis to evaluate if there is relationship between changes in the skin color of beans and the phenomenon "hard-to-cook beans." The results showed that this relationship exists and that the proposed model can be used to detect hard-to-cook beans.

All the five works above (Kiliç et al. (2007), Aguilera et al. (2007), Venora et al. (2007, 2009) and Laurent et al. (2010)) demonstrate the importance of computer vision systems for inspection of beans. However, the systems developed in these works do not spatially localize the grains. In addition, the proposed approaches for segmentation fail in images with "glued" grains. For this reason, in these works, the grains are placed purposely spaced from each other before acquiring the image to facilitate the segmentation or only previously segmented grains are processed. This is a very severe limitation that hinders the applicability of such systems in industrial processes. 


\subsection{The problem and main contributions}

In this work, we propose a computer vision system that spatially localizes each bean grain and classifies it as Carioca, Mulatto or Black (Fig. 1). This is a practical problem, because Carioca is the most consumed bean variety in Brazil, dominating $70 \%$ of market share. The Black accounts for $20 \%$ of market share and all other varieties together totalize 10\% (Souza et al., 2013). Moreover, Black and Mulatto are the two nonpredominant grains most commonly found in batches of Carioca beans. As we said above, a batch of beans only can be labeled as Carioca if it contains no more than $3 \%$ of Black grains and $10 \%$ of Mulatto grains.

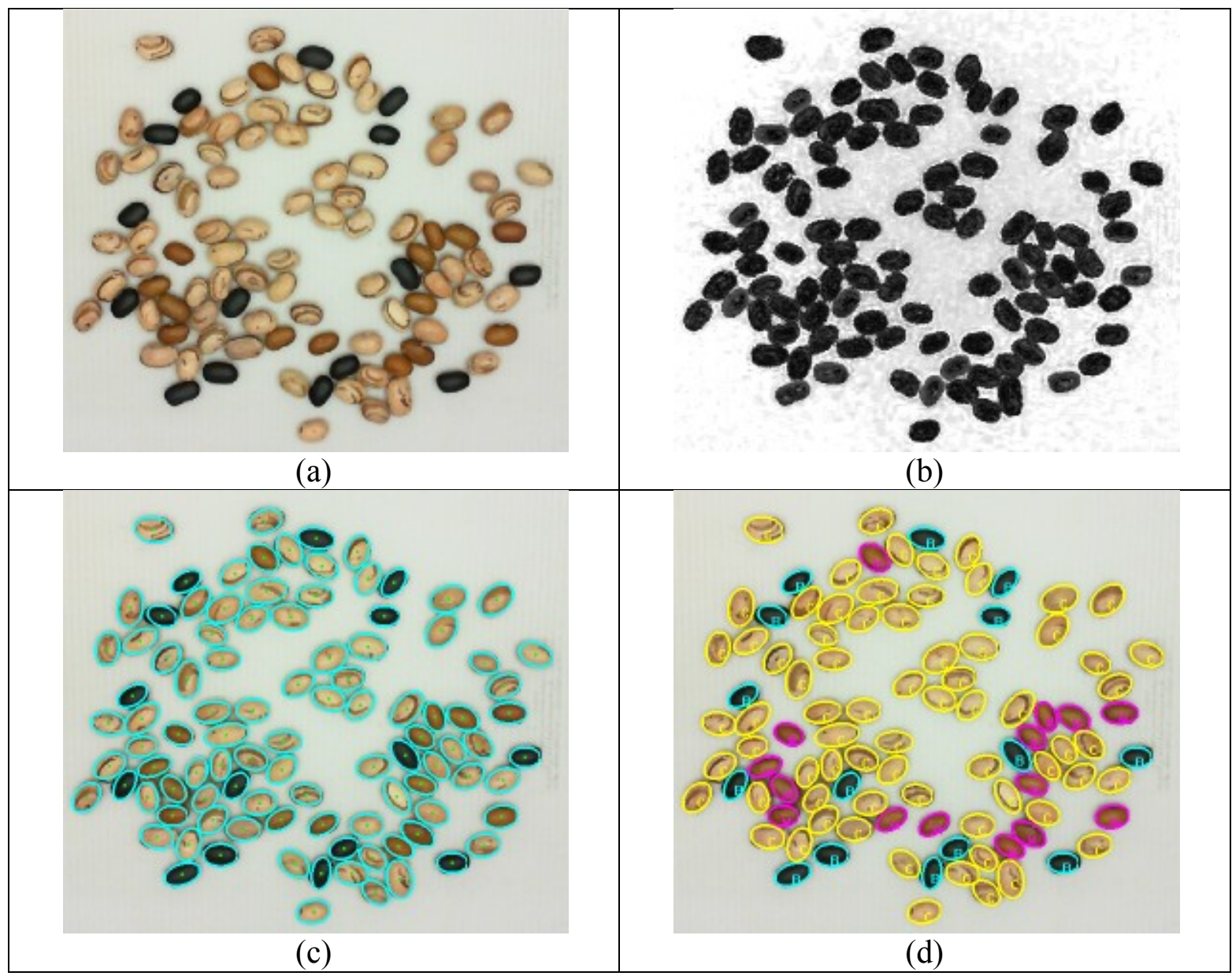

Fig. 2: The three modules of our computer vision system working in "sampling mode". (a) The acquired image. (b) Pixel color mapping to black (foreground) or white (background). (c) Grain segmentation (correlation-based granulometry). (d) Grain classification. 
The image acquisition system makes use of an input box that distributes spatially and temporally the grains over a conveyor belt, a lamp with acrylic plate to illuminate uniformly the beans and a webcam. After the acquisition, a computer vision system processes the acquired image (Fig. 2a). This system is composed of three modules. In the first module, the image pixels are mapped to black (foreground), white (background) or different shades of gray depending on the color of the pixel (Fig. 2b). In the second module, each grain is segmented and spatially localized (Fig. 2c), using the correlation-based multishape granulometry proposed by Kim et al. (2013). In the third step, each grain is classified as Carioca, Mulatto or Black (Fig. 2d).

In our opinion, each of the three modules contains some scientific contribution. However, we think that the main contribution of this paper lies in the second module where we use correlation-based granulometry to single out each bean grains. This technique spatially localizes each grain, together with its size, eccentricity and rotation angle, even if some grains are glued together (Figs. $2 \mathrm{~b}$ and $2 \mathrm{c}$ ), overcoming the shortcoming of other systems aimed at analyzing and classifying seeds and grains. We think that this technique can be used in many other agricultural product inspection systems for segmenting seeds and grains.

Once the grains are precisely localized, many features can be extracted in order to classify them. In this work, we extract features only to classify the grains in three subclasses: Carioca, Mulatto and Black. As future works, we think that it is possible to automatically detect many beans defects (broken, moldy, burned, crushed, chopped, sprouted, wrinkled, stained, discolored, etc.), extracting other features. 


\section{Image Acquisition System}

We built a low-cost prototype composed of a grain input box, a conveyor belt and an image acquisition chamber, as showed in the schematic design (Fig. 3). The beans are loaded in the input box, which spreads the grains in time and space. The dimensions of the conveyor belt are $150 \mathrm{~cm} \times 25 \mathrm{~cm}$. The image acquisition chamber is covered by light-tight paper to eliminate the influence of the external light. It contains a circular fluorescent lamp with translucent acrylic plate for illuminating uniformly the beans and a Microsoft LifeCam HD-5000 for acquiring the images. The velocity of the conveyor belt, the camera and the lamp are controlled by the computer.

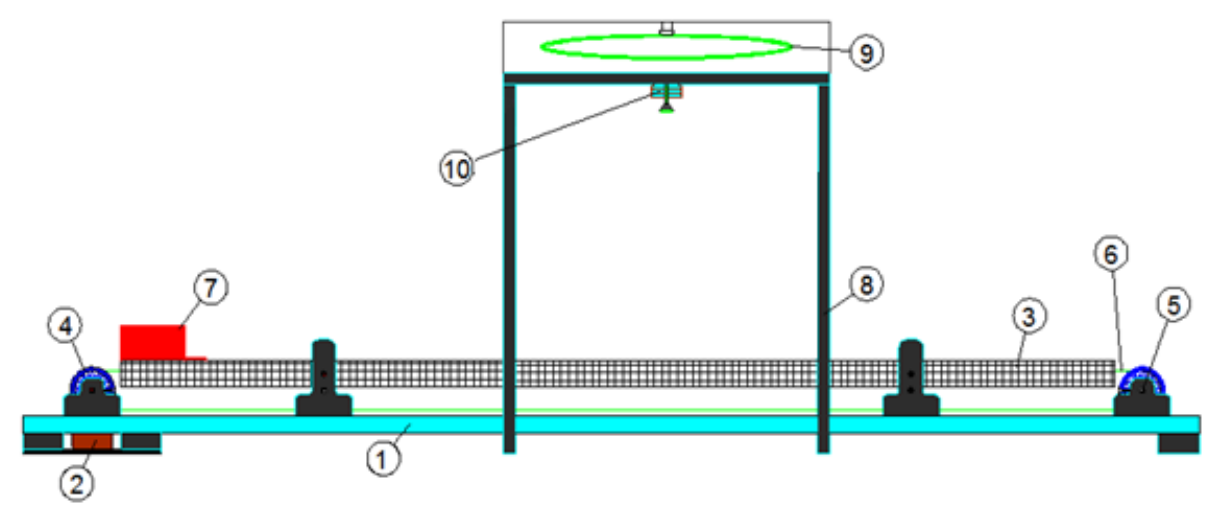

Fig. 3: Schematic design of the apparatus used in experiments: (1) table to support the components; (2) step motor; (3) table to support the conveyor belt; (4) rollers; (5) support bearings; (6) conveyor belt; (7) input box; (8) image acquisition chamber; (9) circular fluorescent lamp; (10) camera.

Although the power consumption of the circular fluorescent lamp is low (15W), we can eliminate it to save energy, using only the ambient light where the prototype is located, and then enhance the acquired image using an appropriate techniques (e.g., Singh et al. 2014; Huang et al., 2013; Cheng et al., 2013; Ding et al., 2014.). However, this could cause 
the loss of some details in the acquired image (e.g. thin streaks present in the grains), decreasing the robustness of the grain classification module.

The prototype (Fig. 4) can operate in two modes: sampling mode or continuous mode. In both cases, the speed of the conveyor belt was approximately $0.02 \mathrm{~m} / \mathrm{s}$.

In the sampling mode, a small quantity of beans (for example, 100 grains) is placed in the input box. In this mode, the exact quantities of each variety of beans are known because they were previously counted, making it possible to determine precisely the error rate. The grains fall automatically from the input box on the conveyor belt, distributing them in space and time. The conveyor belt transports the grains to the image acquisition chamber where the image is acquired. The computer automatically determines the best moment to take the photo.

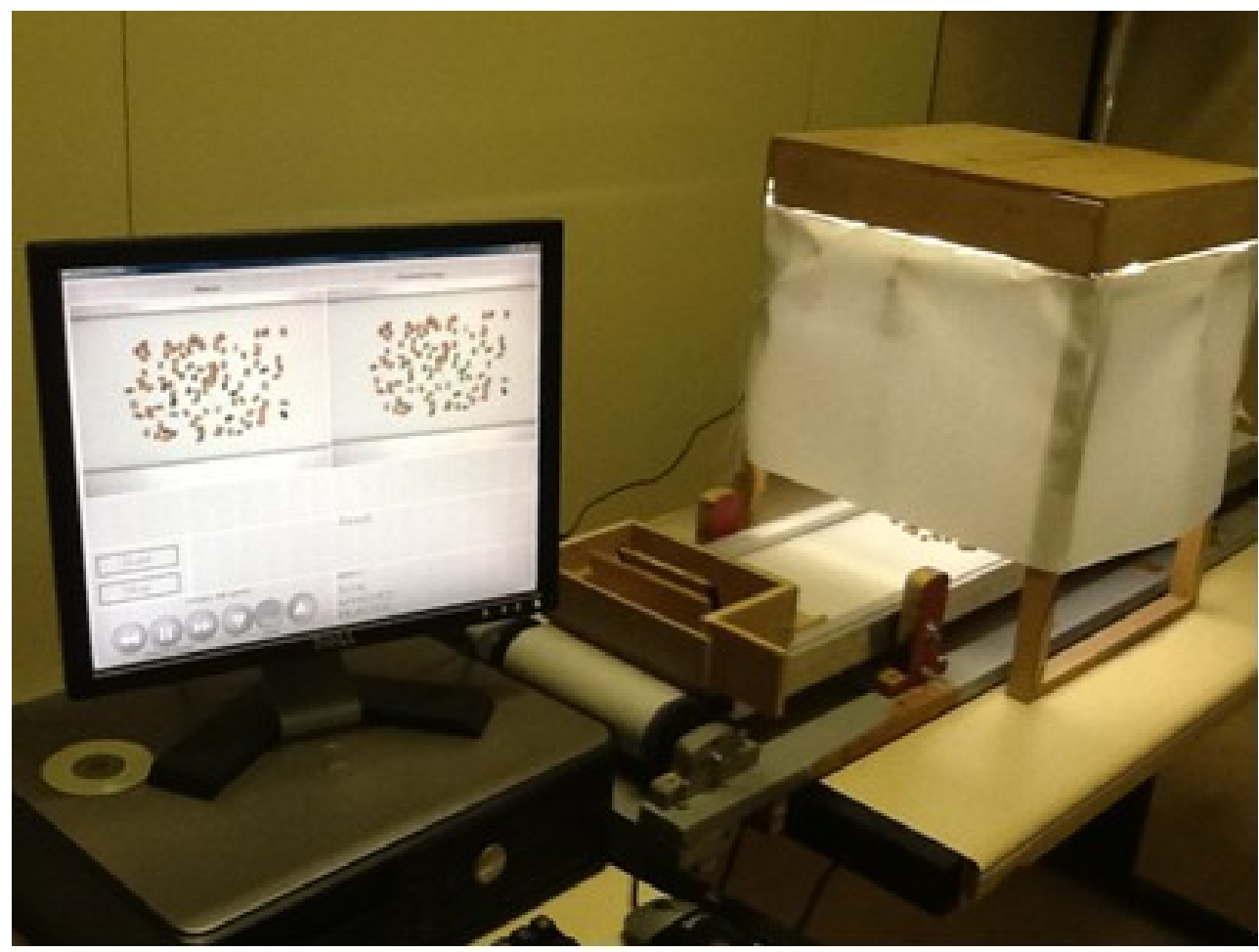

Fig. 4: The apparatus used in the experiments and the interface of the proposed computer vision system. 
In continuous mode, the user can load up to $1 \mathrm{~kg}$ of beans in the input box. The grains automatically and continuously fall from the input box on the conveyor belt. The conveyor belt transports the grains to the image acquisition chamber. The belt stops automatically at the scheduled times for the image acquisition. In this mode, it is not possible to determine the precise error rate of the system, because some grains are missed between two consecutive photos. We prepared mixtures of beans with known proportions of each grain variety. Our system determined experimentally these proportions in continuous mode.

\section{Computer Vision System}

We propose a computer vision system to analyze batches of beans. It is composed of three modules: pixel color mapping, grain segmentation and grain classification. We describe each of them in details in following subsections.

\subsection{Pixel color mapping}

To facilitate the understanding of this module, we describe it twice: first we provide a very simplified overview and then we describe it in details.

Simplified overview: The purpose of this module is to map each pixel of the acquired image (Fig. 2a) to black (beans) or white (background, Fig. 2b). To do it, we use supervised learning. We take some pixels of the beans (positive training data) and some pixels of the background (negative training data). Then, given a pixel $c$ to classify, we search for the most similar color in the training set. We assign the pixel $c$ to the class of the pixel with the most similar color, that is, we use the nearest neighbor learning (1-NN). 
Detailed description: The purpose of this module is to map each pixel of the acquired image (Fig. 2a) to black if the pixel color is a typical color of beans (foreground), and to white if the pixel color is a typical background color (Fig. 2b). When the system cannot decide whether the pixel color is foreground or background, it maps the unknown pixel to different shades of gray according to similarities to known foreground or background colors. This module prepares the acquired image for the grain segmentation in the next module.

First, we extract manually from the sample images some background and bean colors, assuring that almost all possible background colors as well as almost all colors of the different varieties of the beans are well represented (Figs. 5a-5e). Let us denote the set of all sample background colors as $B$ and the set of all sample foreground colors as $F$. Let $c$ be the color of a pixel in the acquired image. Then, we use the following algorithm, based on the $k$-nearest neighbor $(k-\mathrm{NN})$ searching:

1) Find the sample background color $b \in B$ most similar to $c$, that is, $d(b, c) \leq d(x, c), \forall x \in B$, for some distance metric $d$ defined in some color space. We use the Euclidian distance in RGB space, because even this simple metric yields very low error rates. Clearly, more sophisticated color difference metrics can also be used, such as the Euclidean distance in the CIELAB space (Connolly and Fleiss, 1997).

2) Find the sample foreground color $f \in B$ most similar to $c$, that is, $d(f, c) \leq d(x, c)$ ,$\forall x \in F$.

3) Compute the output color $s$ as the linear interpolation of the two distances $s=d(f, c) /[d(f, c)+d(b, c)]$. 
The color mapped image (Fig. 2b) is obtained by repeating this process for each pixel of the acquired image (Fig. 2a).

(a)

(b)

(c)

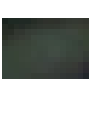

(d)

(e)

Fig. 5: Some of the color samples used in pixel color mapping. (a) Background color sample. (b)-(e) Bean color samples.

\subsection{Grain segmentation}

After the pixel color mapping, each grain must be localized spatially, together with its size, eccentricity and rotation angle, even if some grains are glued together (Figs. 2b and 2c). For this task, we use correlation-based granulometry proposed by Kim et al. (2013). The present work is the first that uses correlation-based granulometry for analyzing seeds or grains. We think that this is the main contribution of this paper, because other papers that analyze seeds and grains usually fail to individualize glued grains.

Granulometry is the process of measuring the size distribution of different objects/grains in a granular material. The size distribution (also known as granulometric curve or pattern spectrum) is the histogram of objects as function of radius. Usually, the granulometry is based on mathematical morphology (Dougherty et al., 1992) or in edge detection (Maerz et al., 1996). Both present shortcomings: mathematical morphologybased granulometry does not localize explicitly each grain, making it impossible to classify the grains; edge-based granulometry fails if there is no clear edge, like in glued grains.

Kim et al. (2013) have proposed a different approach for the granulometry, based on a simple idea: Compute the correlations with kernels that represent all the shapes, eccentricities, orientations and sizes of the grains (Fig. 6). Correlation has been used for a 
long time to find templates in images, in a process named template matching (Lewis, 1995). So, computing the correlations between the image and the kernels that represent all possible shapes of beans corresponds to finding all these shapes in the image. The correlation peaks represent the localizations of the grains. Unfortunately, this process detects many false grains together with the true grains. To filter out the false detections, the peaks with low correlation and peaks that have large intersections with other peaks are discarded. We describe this process in more details below.

The discrete cross correlation between two real-valued images $T$ and $A$ is defined:

$$
C(x, y)=T(x, y) \circ A(x, y)=\sum_{i} \sum_{j} T(i, j) A(x+i, y+j) .
$$

In practice, images are defined only inside a rectangular domain. An image is considered to be filled with zeros outside of its domain. It is well-known that cross correlation can be efficiently computed using FFT (Fast Fourier Transform - see some textbook on image processing for more details, for example, Gonzalez and Woods, 2002). In our case, we will use cross correlation to find a (usually small) template image $T$ inside a (usually large) image to analyze $A$, an operation known as template matching, and this operation is accelerated using FFT.

Fig. 6 present the set of all $n=162$ kernels $T_{i}$ used to localize the beans. We used elliptical kernels with three eccentricities, three scales, rotated in 18 angles. This set of kernels contains all possible shapes of the beans. If you want (for example) find smaller grains, smaller kernels should be included in the set. If you want to find (for example) more elongated grains, kernels with higher eccentricity should be included in the set. 
Fig. 6: Kernels used localize the grains.

In each kernel, the number of black pixels (with negative values) is nearly equal to the number of white ones (with positive values). Gray pixels are zeros. Moreover, in each kernel the sum of all negative pixels is -0.5 and the sum of all positive pixels is +0.5 . As the grayscales of the color mapped image $A$ (Fig. 2b) ranges from 0 (black) to 1 (white), the correlation image $T \circ A$ will range from -1 to +1 . Let us denote the images resulting from the cross correlation between the acquired image $A$ and kernel $T_{i}$ as $C_{i}$, that is:

$$
C_{i}(x, y)=T_{i}(x, y) \circ A(x, y), \quad 1 \leq i \leq n
$$

Let us define the pixelwise maximum of the correlation images as:

$$
C(x, y)=\stackrel{n}{M A} X\left[C_{i}(x, y)\right]
$$

A pixel $(x, y)$ is a peak in $C$ if it is greater than or equal to its eight spatial neighbor pixels. Each peak $C(x, y)$ possibly corresponds to a bean grain in $A$. The scale, eccentricity and angle of the detected grain are given by the corresponding parameters of the kernel. Unfortunately, this process detects many non-existing grains together with the real grains. We filter out the false grains using two parameters chosen by the user:

The first parameter $\tau(0 \leq \tau \leq 1)$ is the correlation threshold parameter. The peaks with correlations lower than $\tau$ are discarded. In our initial experiment, we used $\tau=0.18$. 
The second parameter $\gamma(0 \leq \gamma \leq 1)$ controls the allowed amount of intersection between the grains. If $\gamma=0$, the grains cannot intersect each other in the slightest. If $\gamma=1$, a grain can lie completely inside another grain. In our application, we used $\gamma=0.1$, meaning that up to $10 \%$ of the area of a bean can intersect another bean.

We initially chose the parameters $\tau$ and $\gamma$ based on "common sense" and to minimize errors in a small set of images. We then applied the segmentation using the chosen parameters in the remaining images. The same procedure can be used to choose the appropriate parameters in a real application.

Our algorithm sorts and scans the correlation peaks in decreasing correlation order, discarding a grain $O_{1}$ with correlation $c_{1}$ if there is some other grain $O_{2}$ with correlation $c_{2}>c_{1}$ that satisfies:

$$
\operatorname{area}\left[O_{1} \cap O_{2}\right]>\gamma \operatorname{area}\left[O_{2}\right]
$$

Using this technique in the initial experiment with 100 images and 10,000 grains, our system missed two grains, detected two grains as if they were a single grain, and detected a nonexistent grain. Figure 7 depicts 3 out of these 4 errors.

If the grains are so glued together that the background becomes almost invisible then our technique will fail to segment the grains. In this case, the color mapping module will map almost all pixels to black. Clearly, it is impossible to segment an almost completely black image. However, the images depicted in this paper were obtained by an automated process, so it is possible to spread the grains mechanically as shown in our paper or in an even more dispersed way. 


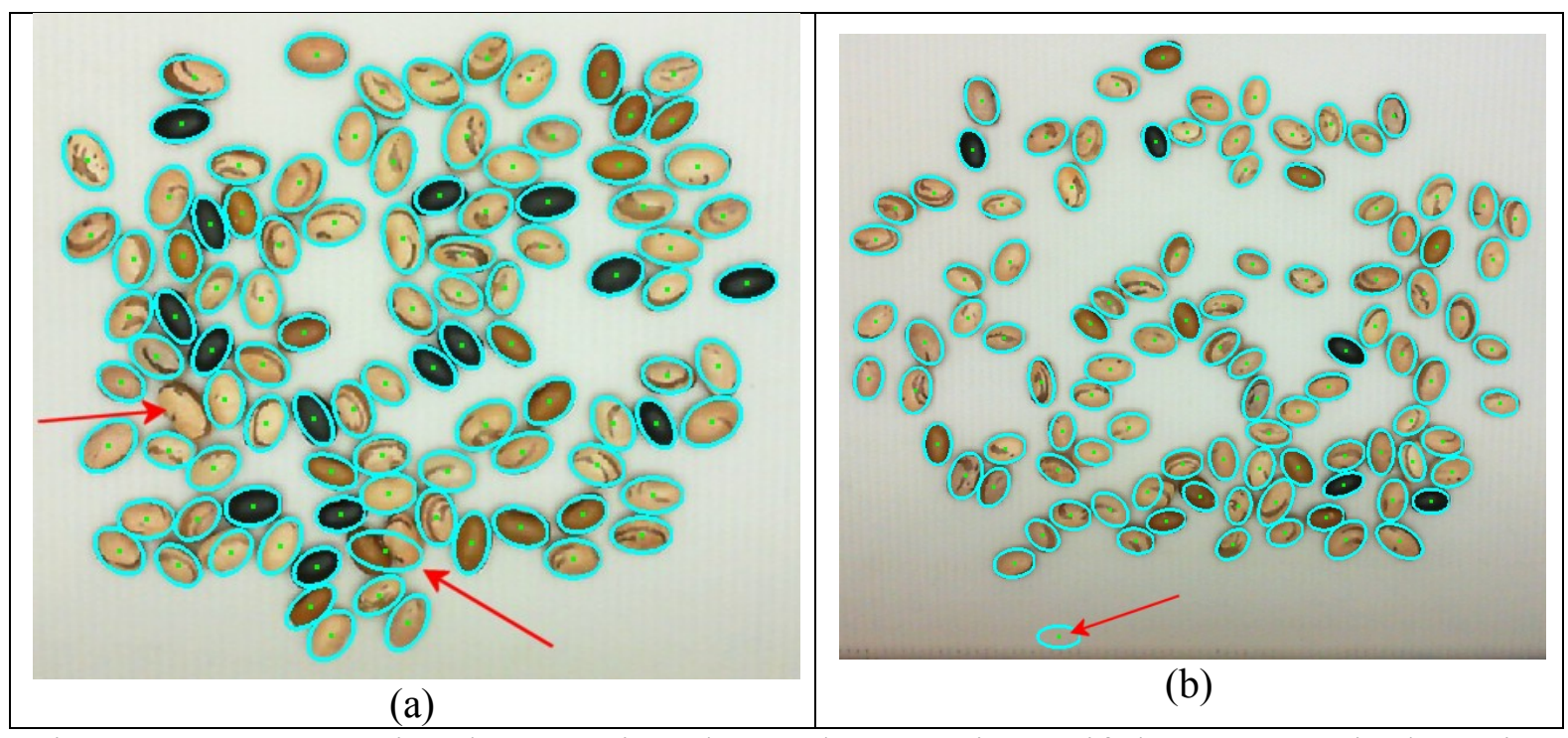

Fig. 7: Our system missed two grains, detected two grains as if they were a single grain, and detected a nonexistent grain, in the initial experiment searching for 10000 grains. Three out of these four errors are depicted above.

\subsection{Grain classification}

After spatially localizing each grain, it must be classified as Carioca, Mulatto or Black. We use as feature only the "predominant color" of each grain in the RGB color space. Clearly, other features (as textures) can also be used. However, we did not test them because even the chosen simple feature yielded the desired results. Our grain classification module was designed only to classify the three varieties of beans according to the predominant color. If the user wants to classify other types of grains, the proposed algorithm should be appropriately adjusted.

Fig. 8a depicts a typical segmented Carioca bean. First, the outer pixels of the segmented images are discarded, shrinking the elliptical kernel by 0.8 , to assure that the segmented bean does not contain any background color (Fig. 8b).

A segmented Black bean (Fig. 8c) can be easily recognized, because its predominant color is very different from the colors of Carioca or Mulatto beans. However, it is not easy to distinguish a Carioca bean from a Mulatto bean. The predominant color of a Cario- 
ca bean is beige but most Carioca grains have brown streaks (Fig. 8b). The dominant color of a Mulatto bean is reddish brown (Fig 8d), but some grains may have streaks on beige. The color of brown streaks of Carioca beans is very similar to the predominant color of Mulatto bean and, sometimes, these streaks occupy a considerable area of the bean (Fig. 8e). Likewise, some parts of a Mulatto beans may have color very similar to the color of a Carioca bean (Fig. 8f).

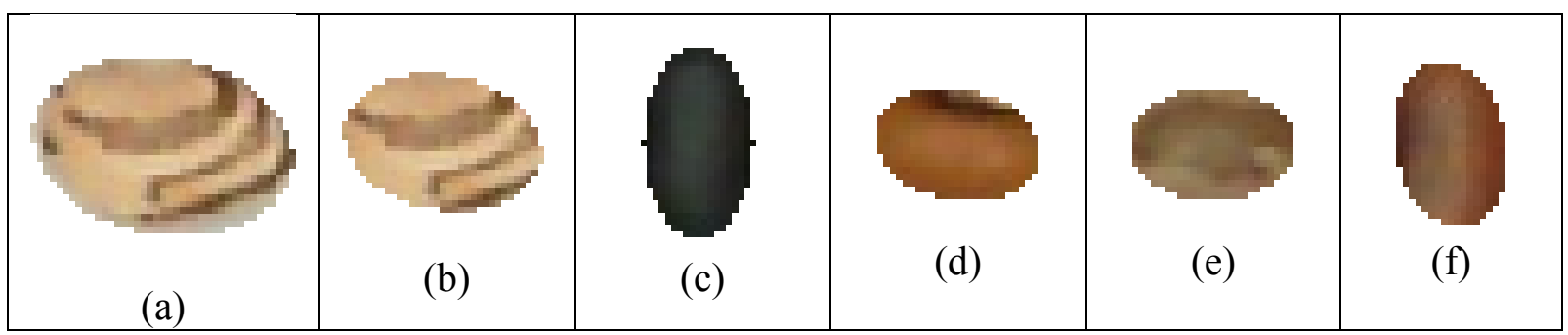

Fig. 8: Examples of segmented beans. (a) A typical Carioca bean. (b) The same bean, removing the pixels located near the border. (c) A typical Black bean. (d) A typical Mulatto bean. (e) An atypical Carioca bean with the brown streaks occupying most of the grain area. (f) An atypical Mulatto bean with grayish brown color that resembles Carioca bean.

In general, a bean grain $G$ may have one or two colors. The following algorithm detects the two representative colors of the grain $G$. If these two colors are similar, we conclude that the grain $G$ has only one color. If they are dissimilar, we take the lighter color as the predominant color. First, we create the palette color set $P$ with all colors that occur in the grain $G$. Then, we separate $P$ in two color sets $C_{1}$ and $C_{2}$ (so that $P=C_{1} \cup C_{2}$ ) using the $k$-means algorithm, with $k=2$ (MacQueen, 1967). Let $f_{1}$ and $f_{2}$ be the relative frequencies of $C_{1}$ and $C_{2}$, that is, $f_{1}=\# C_{1} /\left(\# C_{1}+\# C_{2}\right)$ and $f_{2}=\# C_{2} /\left(\# C_{1}+\# C_{2}\right)$, where $\#$ indicates the cardinality of the set. Let $c_{1}$ and $c_{2}$ be the geometric centers of $C_{1}$ and $C_{2}$. Let us suppose, without loss of generality, that $f_{1} \geq f_{2}$. Then, the following algorithm is used to determine the predominant color of the grain $G$ : 


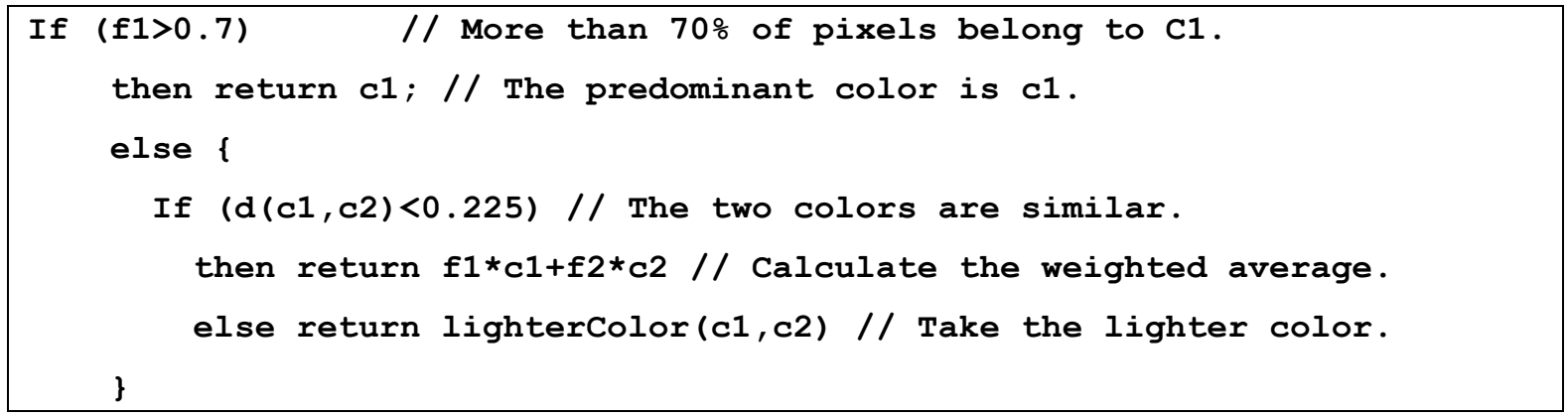

As before, $d\left(c_{1}, c_{2}\right)$ is the Euclidean distance in the RGB space. The parameters 0.7 and 0.225 were chosen experimentally to minimize the errors. This algorithm does:

1) Using the $k$-means algorithm, the pixels of the grain $G$ are divided in classes $C_{1}$ and $C_{2}$, with centers $c_{1}$ and $c_{2}$. Let us suppose that $C_{1}$ is more frequent than $C_{2}$.

2) If more than $70 \%$ of the pixels belong to $C_{1}$, then $c_{1}$ is chosen as the predominant color.

3) Else if the colors $c_{1}$ and $c_{2}$ are similar, then probably the grain $G$ has a single color (as the grain in Fig. 8c). In this case, the weighted mean of colors $c_{1}$ and $c_{2}$ is chosen as the predominant color.

4) Else (if the two colors $c_{1}$ and $c_{2}$ are dissimilar) the lighter color is chosen as the predominant color, because the lighter color of a Carioca bean is very different from the lighter color of a Mulatto bean.

After extracting the predominant color, it is necessary to classify the grain as Carioca, Mulatto or Black. For this task, we use again the $k$-nearest neighbor searching. In the initial experiment, we took 71 Carioca beans, 17 Mulatto beans and 15 black beans as the training samples. Then, we computed their predominant colors. Given a grain $G$ to be clas- 
sified, we compute its predominant color and search for the sample grain $S$ with the most similar color. The test grain $G$ receives the same classification as the grain $S$.

Using this algorithm, our system made one single mistake in the initial experiment when classifying 9996 grains (we are disregarding those grains that were incorrectly localized in the grain segmentation module). Fig. 9 illustrates the only misclassified grain. By a rare chance, the brown streaks dominate the view of this Carioca bean, making it look like a Mulatto bean. Clearly, other more sophisticated machine learning algorithms (such as Multilayer Perceptron, Support Vector Machine, Adaboost, etc.) can be applied for the same task. However, we did not test them because even the simple $k$-NN learning yielded very high success rate.

In spite of the light-tight paper used in our acquisition system, we noted that the "predominant color" changes with the external lighting. Thus it is very important to keep the illumination constant for the classifier to be successful.

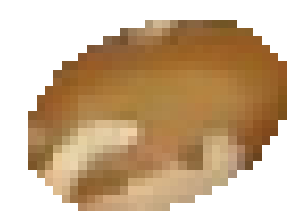

Fig. 9: The Carioca grain classified erroneously as Mulatto. Note that brown streaks predominates the view. Our system made this single mistake when classifying 9996 grains in the initial experiment. 


\section{Initial experiment (sampling mode experiment 1)}

The computer vision system proposed in this work was implemented in $\mathrm{C} / \mathrm{C}++$ using image processing and computer vision libraries Ceikeikon ${ }^{2}$ and $\mathrm{OpenCV}^{3}$. In our experiments, we took into account the three most consumed beans in Brazil: Carioca, Mulatto and Black beans. We purposely "contaminated" batches of Carioca beans with Mulatto and Black grains, because we wanted simulate the detection of non-predominant varieties in batches of Carioca beans. We evaluated 100 images of beans, each one containing 100 grains. These images were divided into 10 subsets, according to the percentage of nonpredominant grains (Table 1).

Table 1: Image set used in the initial experiment.

\begin{tabular}{|c|c|c|c|c|}
\hline Subset & Total of images & $\begin{array}{c}\text { Number of Carioca } \\
\text { beans in each image }\end{array}$ & $\begin{array}{c}\text { Number of Mulatto } \\
\text { beans in each image }\end{array}$ & $\begin{array}{c}\text { Number of Black } \\
\text { beans in each image }\end{array}$ \\
\hline 1 & 10 & 100 & 0 & 0 \\
\hline 2 & 10 & 95 & 5 & 0 \\
\hline 3 & 10 & 95 & 0 & 5 \\
\hline 4 & 10 & 90 & 5 & 5 \\
\hline 5 & 10 & 85 & 10 & 10 \\
\hline 6 & 10 & 85 & 5 & 10 \\
\hline 7 & 10 & 80 & 15 & 0 \\
\hline 8 & 10 & 85 & 0 & 15 \\
\hline 9 & 10 & 85 & 15 & 15 \\
\hline 10 & 10 & 70 & $\mathbf{6 5 0}$ & $\mathbf{6 5 0}$ \\
\hline Total & $\mathbf{1 0 0}$ & $\mathbf{8 7 0 0}$ & & \\
\hline
\end{tabular}

The obtained results have already been described above. The system localized correctly 9996 out of 10000 grains and classified correctly 9995 out of 9996 grains.

\footnotetext{
${ }^{2}$ Available at http://www.lps.usp.br/ hae/software/

${ }^{3}$ Available at http://opencv.org/
} 
In a Intel Core i7 $2.2 \mathrm{GHz}$ computer, our system typically takes 18 s to process an image with $800 \times 600$ pixels $(6 \mathrm{~s}$ to map the pixel colors, $11 \mathrm{~s}$ to segment the grains and $1 \mathrm{~s}$ to classify the grains), without using multi-threading or special optimization techniques.

\section{More experiments}

To assure that the results obtained in the initial experiment are reliable, we made other experiments using other batches of beans, both in sampling and continuous modes. We bought Carioca, Mulatto and Black beans from different trade brands, mixed them and used the resulting mixtures in the experiments. We changed some parameters from the initial experiment, because the new parameters resulted in fewer errors:

a) In the grain segmentation, we changed the correlation threshold parameter from $\tau=0.18$ to $\tau=0.10$.

b) In the grain classification, we changed the threshold used to decide whether two colors are similar from 0.225 to 0.05 .

We use these new parameters in all the following experiments.

\subsection{Sampling mode experiment 2}

In this experiment, we used similar number of the three varieties of beans. Table 2 shows the number of each variety of beans used in this experiment. We used the 300 beans extracted from 3 images of this set as the training samples for the classification. There were 3 segmentation errors (two missed beans and a bean that was detected as two) and 11 classification errors. In all misclassifications, a Mulatto bean was mistaken for a Carioca bean or vice-versa. No misclassification involved Black beans. 
Table 2: Number of beans varieties in sampling mode experiment 2.

\begin{tabular}{|c|c|c|c|c|}
\hline Subset & Total of images & $\begin{array}{c}\text { Number of Carioca } \\
\text { beans in each image }\end{array}$ & $\begin{array}{c}\text { Number of Mulatto } \\
\text { beans in each image }\end{array}$ & $\begin{array}{c}\text { Number of Black } \\
\text { beans in each image }\end{array}$ \\
\hline 1 & 10 & 20 & 20 & 60 \\
\hline 2 & 10 & 20 & 60 & 20 \\
\hline 3 & 10 & 60 & 20 & 20 \\
\hline 4 & 10 & 40 & 40 & 20 \\
\hline 5 & 10 & 40 & 20 & 40 \\
\hline 6 & 10 & 20 & 40 & 40 \\
\hline 7 & 10 & 30 & 30 & 40 \\
\hline 8 & 10 & 30 & 40 & 30 \\
\hline 9 & 10 & 40 & 30 & 30 \\
\hline 10 & 10 & 34 & 33 & 33 \\
\hline Total & $\mathbf{1 0 0}$ & $\mathbf{3 3 4 0}$ & $\mathbf{3 3 3 0}$ & $\mathbf{3 3 3 0}$ \\
\hline
\end{tabular}

\subsection{Sampling mode experiment 3}

In this experiment, we made a new image acquisition using new beans but using the same number of the three varieties used in the initial experiment and described in Table 1. There was no segmentation error. However, there were 25 classification errors. Again, no misclassification involved Black beans. We used the 300 beans extracted from 3 images of this set as the training samples for the classification.

\subsection{Summary of sampling mode experiments}

We made three experiments in sampling mode, each one with 10,000 beans. Our system made $4+3$ segmentation errors. Considering $2+2$ false positives (FP) and $4+2$ false negatives $(\mathrm{FN})$, we have recall rate (or true positive rate or hit rate):

$$
\text { recall }=T P /(T P+F N)=29993 /(29993+6)=99.980 \%
$$

and precision rate (or positive predictive value):

$$
\text { precision }=T P /(T P+F P)=29993 /(29993+4)=99.987 \% .
$$


It is also possible to compute other metrics. For example, $F_{1}$ metric and Similarity measure (Maddalena and Petrosino, 2008; Huang and Chen, 2014):

$$
F_{1}=\frac{2 \times \text { Recall } \times \text { Precision }}{\text { Recall }+ \text { Precision }}=99.983 \%
$$

$$
\text { Similarity }=\frac{T P}{T P+F N+F P}=\frac{29993}{29993+6+4}=99.967 \%
$$

Our system made $1+11+25=37$ classification errors when classifying 29,993 grains (we are disregarding those grains that were incorrectly localized in the grain segmentation module), yielding $99.877 \%$ of classification success rate outperforming the rates of $90.6 \%$, 99.56\% and 98.49\% achieved by of Kiliç et al. (2007), Venora et al. (2007) and Venora et al. (2009), respectively. However, we emphasize that the conditions of our experiments are different from these works. The system made considerably more classification errors in experiments 2 and 3 than in the initial experiment, probably because we used a mixture of beans from many brands in experiments 2 and 3 .

\subsection{Continuous mode experiment 1}

We prepared a mixture of 930 Carioca beans and 70 Black beans, loaded them in the input box, turned on the image acquisition system and acquired the images in continuous mode. We repeated the image acquisition process three times, using the same 1000 beans. In each acquisition, 9 or 10 images were acquired. Each acquisition process (acquiring roughly 10 images with around 1000 beans) takes approximately 4.5 minutes. The total number of grains obtained at each acquisition is less than 1000 because there is always a "gap" between two consecutive photos. We processed these images, obtaining the results

listed in Table 3. For the beans classification, we used the same training samples used in 
"sampling mode experiment 3", removing the Mulatto beans (absent in this experiment).

The obtained percentages of the Black beans are very close to the true percentage $(7 \%)$.

Table 3: Number of beans detected in continuous mode experiment 1.

\begin{tabular}{|r|r|r|r|r|}
\hline & Carioca & Black & $\begin{array}{r}\text { Percentage of } \\
\text { Black }\end{array}$ & Total \\
\hline Acquisition 1 & 893 & 68 & $7.075 \%$ & 961 \\
\hline Acquisition 2 & 914 & 69 & $7.019 \%$ & 983 \\
\hline Acquisition 3 & 894 & 68 & $7.069 \%$ & 962 \\
\hline Total & 2701 & 205 & $7.054 \%$ & 2906 \\
\hline
\end{tabular}

\subsection{Continuous mode experiment 2}

We prepared a mixture of 400 Carioca, 300 Mulatto and 300 Black beans, loaded them in the input box, turned on the image acquisition system and acquired the images in continuous mode. We repeated the image acquisition process three times, using the same 1000 beans. We processed these images, obtaining the results listed in Table 4. The obtained percentages are very close to the true ones $(40 \%, 30 \%$ and $30 \%)$. For the beans classification, we used the same 300 training samples used in "sampling mode experiment 3". Figure 10 depicts the intermediary images generated by our system in continuous mode. Note that the grain segmentation automatically discards the partially occluded grains localized at left and right borders of the image.

Table 4: Number of beans detected in continuous mode experiment 2.

\begin{tabular}{|r|r|r|r|r|r|r|r|}
\hline & \multicolumn{2}{|c|}{ Carioca } & \multicolumn{2}{c|}{ Mulatto } & \multicolumn{2}{|c|}{ Black } & Total \\
\hline Acquisition 1 & 371 & $39.98 \%$ & 280 & $30.17 \%$ & 277 & $29.85 \%$ & 928 \\
\hline Acquisition 2 & 372 & $41.24 \%$ & 263 & $29.16 \%$ & 267 & $29.60 \%$ & 902 \\
\hline Acquisition 3 & 358 & $39.34 \%$ & 281 & $30.88 \%$ & 271 & $29.78 \%$ & 910 \\
\hline Total & 1103 & $40.26 \%$ & 822 & $30.00 \%$ & 815 & $29.74 \%$ & 2740 \\
\hline
\end{tabular}




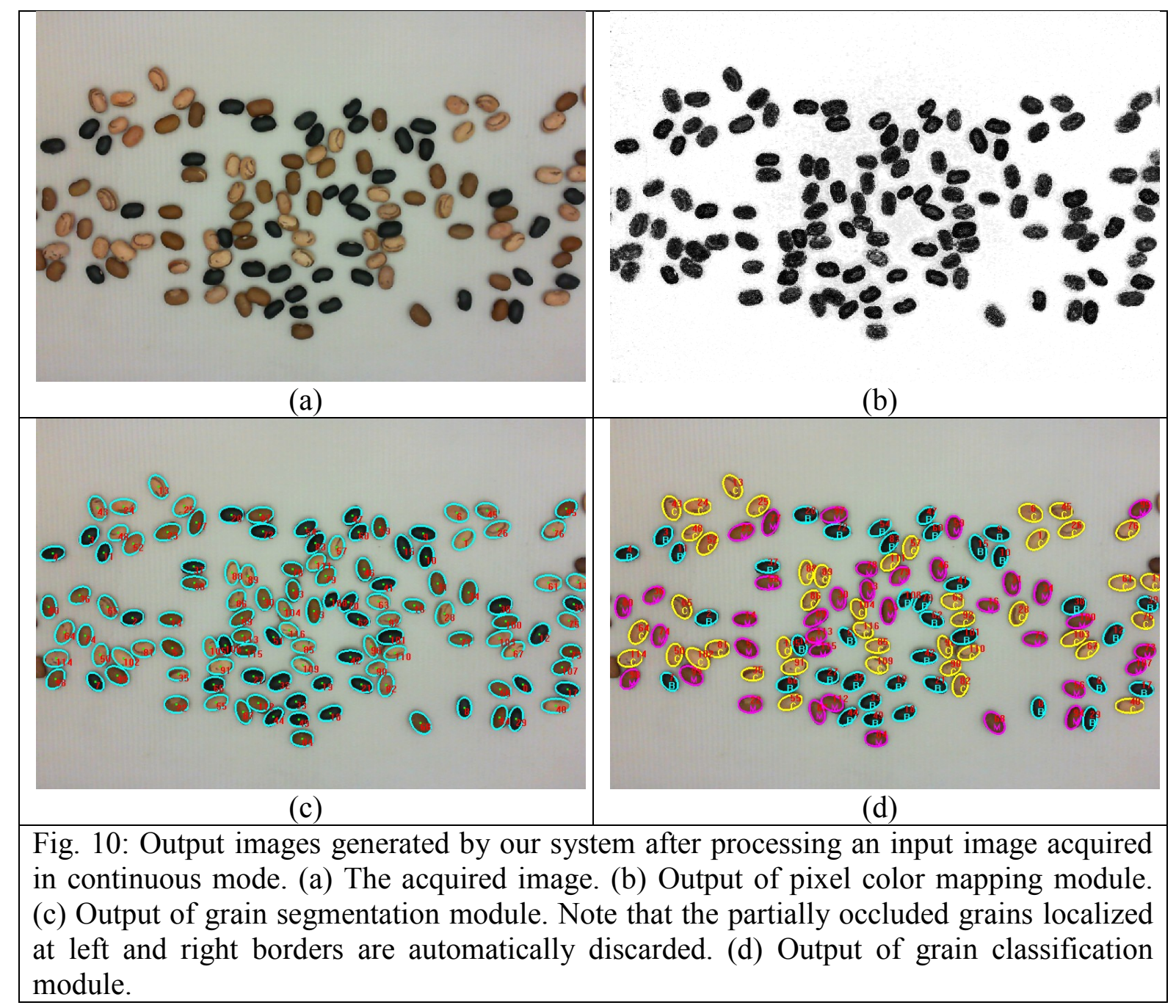

\subsection{Acquiring images without conveyor belt}

We emphasize that our system is designed to replace humans that classify samples of beans, and not to classify the entire batch of beans. So, the image acquisition and processing do not necessarily have to be "super fast". For small quantities of beans, a simple cardboard box (Fig. 11a) may replace the image acquisition system described in Section 2. In this case, the user loads the cardboard box with a sample of beans, shakes the box to spread the beans and takes the photo with uniform illumination (Fig. 11b). The remainder 
of the processing is the same. Certainly, this process is much faster than counting manually the three varieties of beans.

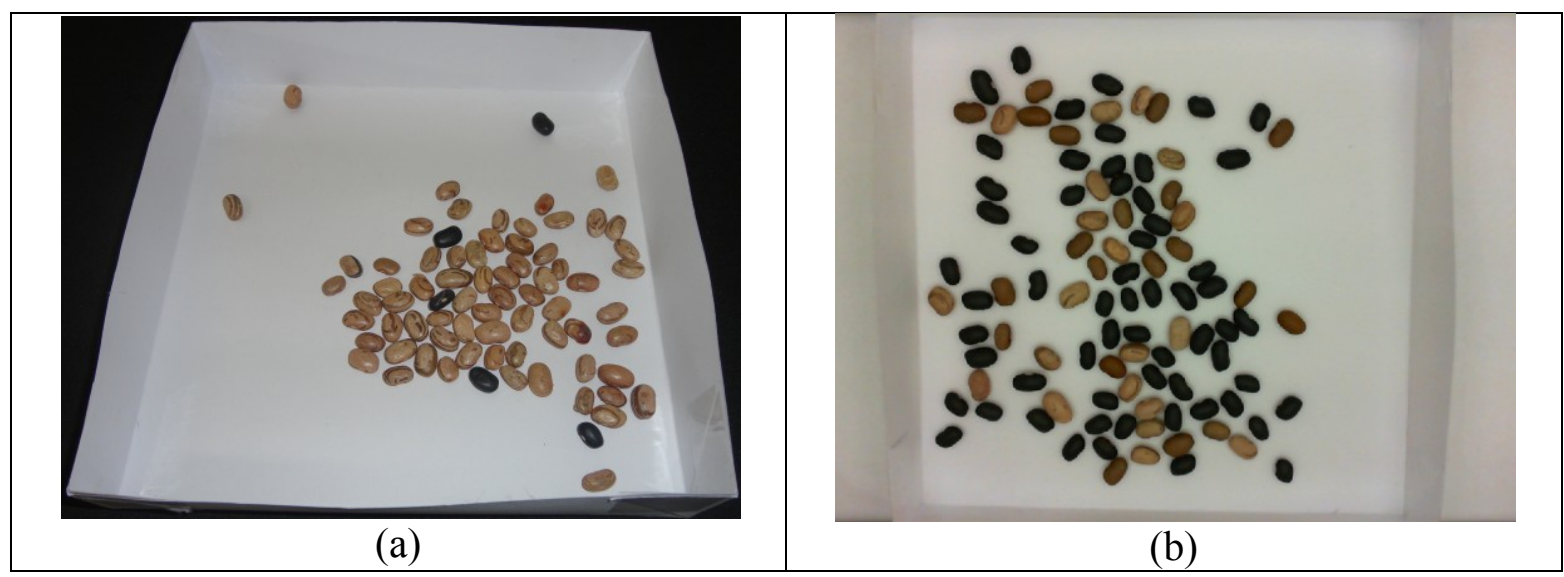

Fig. 11: For small quantities of beans, a simple cardboard box may replace the image acquisition system.

\subsection{Impurities}

We describe in this subsection the behavior of the system in the presence of some "impurities" (unexpected or expected). In all the experiments, we took photos with 34 Carioca, 33 Mulatto, 33 Black beans and 5 "impurities".

Corn grain: We acquired 10 images, each one with 5 corn grains. We processed these images as usually. The system localized correctly 105 grains in all 10 images (both beans and corns). Without feeding the classification module with the corn colors, it classified almost all corn grains as Carioca, because the Carioca color is the most similar to corn color. Feeding the classification module with five corn grain samples, the system classified correctly 41 corn grains and erred 9 (Fig. 12a).

Broken Carioca beans: We acquired 10 images, each one with 5 broken Carioca beans. We processed these images as usually. The system localized correctly 105 beans in all 10 images, even the broken ones. The system classified almost all broken Carioca grains as Carioca. Feeding the classification module with five broken Carioca beans, the 
system classified correctly only 15 broken Carioca grains (erred 35). We conclude that broken Carioca beans can be easily localized, but cannot be distinguished from whole Carioca beans by the classification module. It is necessary to include other features especially designed to classify them.

Peas: We acquired 10 images, each one with 5 peas. We processed these images as before. The system localized correctly 105 grains in all 10 images. Without feeding the classification module with the pea colors, it classified almost all peas as Mulatto beans. Feeding the classification module with five pea samples, the system classified correctly 45 peas grains and erred 5 (Fig. 12b).

Straw: We acquired 10 images, each one with 5 straws. We processed these images as usually. The system localized many non-existing grains in each straw, detecting in average 112.1 grains in each image (Fig. 5c). Straw have color similar to beans. So, the pixel color mapping module cannot distinguish between straw and bean.

Grass: We acquired 10 images, each one with 5 green grasses. Processing these images as usually, the system localizes many non-existing grains in each grass (as in the images contaminated by straws). In average, 106.5 grains were detected in each image. However, as grass is green and none of the three varieties of beans is green, color mapping module can map green color to background. Including some samples of grass in the set of background colors, we detected in average 100.3 grains in each image. That is, the grass was successfully ignored by the system (Fig. 12d). 


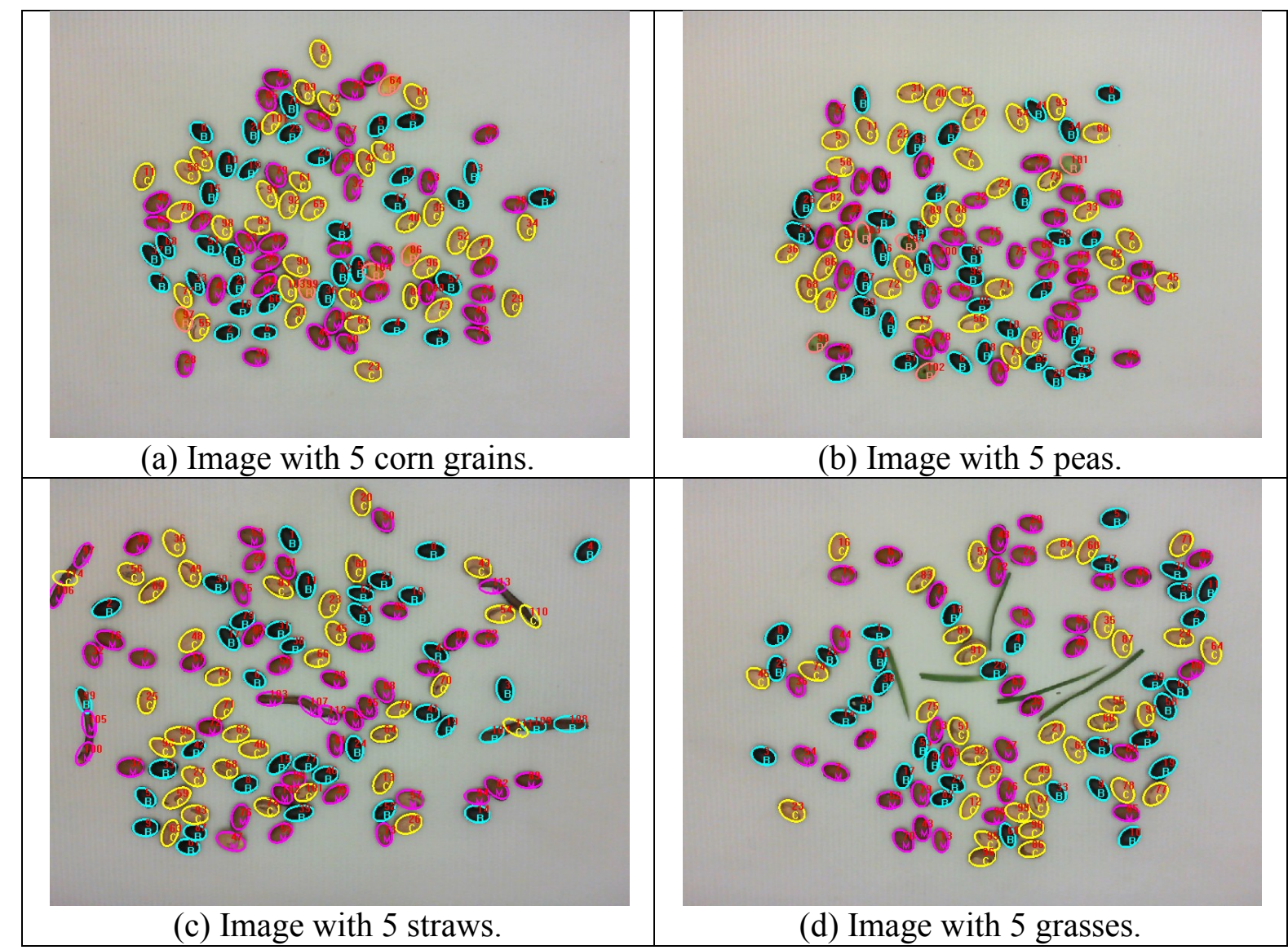

Fig. 12: The behavior of the system in the presence of different "impurities".

\section{Conclusion}

In this work, we have presented a computer system for visual inspection of beans that classifies them according to their skin colors. This system is composed of 3 modules: pixel color mapping, grain segmentation and grain classification. In the color mapping module, we have presented a technique based on $k$-NN learning that maps a pixel to different shades of gray according to its similarity to "beans color" or "background color". In the grain segmentation module, we have used correlation-based granulometry to localize each bean grain, together with its eccentricity, size and rotation angle. In our opinion, this is the most important scientific contribution of this work. This technique yielded recall rate of $99.98 \%$ and precision rate of $99.99 \%$. We think that this technique can be used in many other agricultural product inspection systems to analyze seeds and grains. In grain classifi- 
cation module, we have used $k$-means and $k$-NN to classify beans according to their predominant skin color, obtaining correct classification rate of $99.88 \%$. These extremely high rates show the robustness of the proposed system and indicate that it can be applied in an industrial process for visual inspection of beans.

\section{Acknowledgments}

The authors would like to thank CAPES - Coordination for the Improvement of Higher Education Personnel for the scholarship granted to J. H. Pessota; CNPq - Brazilian National Research Council for the research scholarship granted to H. Y. Kim (Process 302917/ 2012-8) and FAPESP - São Paulo Research Foundation by financial support (Process 2014/ 09194-5).

\section{References}

Aggarwal, A.K., Mohan, R., 2010. Aspect ratio analysis using image processing for ricegrain quality. International Journal of Food Engineering, 6 (5), 1-14.

Aguilera, J.M., Cipriano, A., Eraña, M., Lillo, I., Mery, D., Soto, A., 2007. Computer Vision for Quality Control in Latin American Food Industry: a Case Study. In: Proceedings of 7th Int. Conf. on Computer Vision: Workshop on Computer Vision Applications for Developing Countries, Rio de Janeiro, 1-11.

Alfatni, M.S., Shariff, A.R.M., Abdullah, M.Z., Saeed, O.M.B., Ceesay, O.M., 2011. Recent methods and techniques of external grading systems for agricultural crops quality inspection - review. International Journal of Food Engineering, 7 (3), 1-40. 
Anami, B. S., Savakar, D. G., 2010. Influence of light, distance and size on recognition and classification of food grains' images. International Journal of Food Engineering, 6 (2), 121.

Blasco, J., Aleixos, N., Cubero, S., Juste, F., Gómez-Sanchís, J., Alegre, V., Moltó, E., 2009. Computer vision developments for the automatic inspection of fresh and processed fruits. In: Proceedings of 1st International Workshop on Computer Image Analysis in Agriculture held in Potsdam, Germany, 21-28.

Blasco, J., Cubero, S., Gómez-Sanchís, J., Mira, P., Moltó, E., 2009. Development of a machine for the automatic sorting of pomegranate (Punica granatum) arils based on computer vision. Journal of Food Engineering, 90 (1), 27-34.

BMALS - Brazilian Ministry of Agriculture, Livestock and Supply. Law n ${ }^{\circ}$ 6.305, Decree $n^{\circ}$ 93.563, of 11/11/86, normative instruction n $n^{\circ}$ 12. Retrieved April 2011 from http://ainfo.cnptia.embrapa.br/digital/bitstream/item/97404/1/comunicadotecnico-211.pdf.

Carrillo, E., Peñaloza, A.A., 2009. Artificial vision to assure coffee-Excelso beans quality. In: Proceedings of the 2009 Euro American Conference on Telematics and Information Systems: New Opportunities to increase Digital Citizenship, New York, USA.

Cheng, F., Huang, S., 2013. Efficient histogram modification using bilateral Bezier curve for the contrast enhancement. IEEE Journal of Display Technology, 9 (1), 44-50.

Connolly, C., Fleiss, T., 1997. A study of efficiency and accuracy in the transformation from RGB to CIELAB color space. IEEE Trans. on Image Processing, 6 (7), 1046-1048.

Ding, X., Sun, L., Luo, Y., 2014. Shell histogram equalization of color images, Elsevier Optik - International Journal for Light and Electron Optics, 125 (13), 3350-3354. 
Dougherty, E. R., Newell J.T., Pelz, J.B., 1992. Morphological texture-based maximumlikelihood pixel classification based on local granulometric moments. Pattern Recognition, 25 (10), 1181-1198.

Gómez-Sanchis, J., Martín-Guerrero, J.D., Soria-Olivas, E., Martínez-Sober, M., Magdalena-Benedito, R., Blasco, J., 2012. Detecting rottenness caused by Penicillium genus fungi in citrus fruits using machine learning techniques. Expert Systems with Applications, 39 (1), 780-785.

Gonzalez, R.C., Woods, R.E., 2002. Digital Image Processing, Second Edition, PrenticeHall.

Huang, S., Cheng, F., Chiu, Y., 2013. Efficient contrast enhancement using Adaptive Gamma Correction with Weighting Distribution. IEEE Transactions on Image Processing, 22(3), 1032-1041.

Huang, S.C., Chen, B.H., 2014. Automatic moving object extraction through a real-world variable-bandwidth network for traffic monitoring systems. IEEE Transactions on Industrial Electronics, 61 (4), 2099-2112.

Kiliç, K., Boyacl, I. H., Köksel, H., Küsmenoglu, I.A., 2007. Classification system for beans using computer vision system and artificial neural networks. Journal of Food Engineering, 78 (3), 897-904.

Kim, H.Y., Maruta, R.H., Huanca, D.R., Salcedo, W.J., 2013. Correlation-based multishape granulometry with application in porous silicon nanomaterial characterization. Journal of Porous Materials, 20 (2), 375-385. 
Laurent, B., Ousman, B., Dzudie, T., Carl, M.F.M., Emmanuel, T., 2010. Digital camera images processing of hard-to-cook beans. Journal of Engineering and Technology Research, 2 (9), 177-188.

Lewis, J.P., 1995. Fast template matching, in Proc. Vision Interface, pp. 120-123.

Liu, J., Yang, W.W., Wang, Y., Rababah, T.M., Walker, L.T., 2011. Optimizing machine vision based applications in agricultural products by artificial neural network. International Journal of Food Engineering, 7 (3), 1-23.

MacQueen, J. B. 1967. Some methods for classification and analysis of multivariate observations. In: Proceedings of 5th Berkeley Symposium on Mathematical Statistics and Probability. University of California Press. 281-297.

Maddalena, L., Petrosino, A., 2008. A self-organizing approach to background subtraction for visual surveillance applications, IEEE Trans. Image Processing, 17 (7), 1168-1177.

Maerz, N.H., Palangio, T.C., Franklin, J.A., 1996. WipFrag image based granulometry system. In: Proceedings of the FRAGBLAST, 5th Workshop on Measurement of Blast Fragmentation, Montreal, 91-99.

Mendoza, F., Aguilera, J.M., 2010. Gloss measurements of raw agricultural products using image analysis. Food Research International, 43 (1), 18-26.

Patel, K.K., Kar, A., Jha, S.N., Khan, M.A., 2012. Machine vision system: a tool for quality inspection of food and agricultural products. Journal of Food Science and Technology, 49 (2), 123-141. 
Patil, N.K., Yadahalli, R.M. Pujari, J., 2011. Comparison between HSV and YCbCr Color Model Color-Texture based Classification of the Food Grains. International Journal of Computer Applications, 34 (4), 51-57.

Pesante-Santana, J.A., Woldstad, J.C., 2000. The quality inspection task in modern manufacturing. In: International Encyclopedia of Ergonomics, W. Karwowski (ed.), Taylor and Francis, London.

Razavi, S.M.A., Bostan, A., Rezaie, M., 2010. Image processing and physico-mechanical properties of basil seed (Ocimum basilicum). Journal of Food Process Engineering, 33 (1), $51-64$.

Rodríguez-Pulido, F.J., Gordillo, B., González-Miret, M. L., Heredia, F.J., 2013. Analysis of food appearance properties by computer vision applying ellipsoids to colour data. Computers and Electronics in Agriculture, 99 108-115

Saraswat, M., Arya, K.V., 2013. Supervised leukocyte segmentation in tissue images using multi-objective optimization technique. Engineering Applications of Artificial Intelligence, Available online 5 October 2013. DOI: 10.1016/j.engappai.2013.09.010.

Savakar, D. G., 2012. Identification and classification of bulk fruits images using artificial neural networks. International Journal of Engineering and Innovative Technology, 1, (3), $36-40$.

Savakar, D.G., Anami, B.S., 2009. Recognition and classification of food grains, fruits and flowers using machine vision. International Journal of Food Engineering, 5 (4), 1-25.

Singh, K., Kapoor, R., 2014. Image enhancement using exposure based sub image histogram equalization. Pattern Recognition Letters, 36 (15), 10-14. 
Souza, T.L.P.O., Pereira, H.S., Faria, L.C., Wendland, A., Costa, J.G.C., Abreu, A.F.B., Dias, J.L.C., Magaldi, M.C.S., Sousa, N.P., Peloso, M.J.D., Melo, L.C., 2013. Common bean cultivars from Embrapa and partners available for 2013. Technical Statement, 211, 1-6. Retrieved December 2013 from

http://extranet.agricultura.gov.br/sislegis/action/detalhaAto.do?method=consultarLegislaca oFederal.

Stegmayer, G., Milone, D.H., Garran, S., Burdyn, L., 2013. Automatic recognition of quarantine citrus diseases. Expert Systems with Applications, 40 (9), 3512-3517.

Ticay-Rivas, J.R., del Pozo-Baños, M., Eberhard, W.G., Alonso, J.B., Travieso, C. M., 2013. Spider specie identification and verification based on pattern recognition of it cobweb. Expert Systems with Applications, 40 (10), 4213-4225.

Venora, G., Grillo, O., Ravalli, C., Cremonini, R., 2009. Identification of Italian landraces of bean (Phaseolus vulgaris L.) using an image analysis system. Scientia Horticulturae, 121 (4), 410-418.

Venora, G., Grillo, O., Ravalli, C., Cremonini, R., Tuscany beans landraces, on-line identification from seeds inspection by image analysis and Linear Discriminant Analysis. Agrochimica, 51 (4-5), 2007, 254-268. 